\title{
Análise de zonas homogêneas em séries temporais de precipitação no Estado da Bahia
}

\author{
Camila da Silva Dourado ( $\left.{ }^{1 *}\right)$; Stanley Robson de Medeiros Oliveira (1,2); Ana Maria Heuminski de Avila (3) \\ (1) Universidade de Campinas (UNICAMP), Faculdade de Engenharia Agrícola, Av. Cândido Rondon, 501, 13083-875 Campinas (SP), Brasil. \\ (2) Embrapa Informática Agropecuária, Av. André Tosello, 209, 13083-886 Campinas (SP), Brasil. \\ (3) UNICAMP, Centro de Pesquisas Meteorológicas e Climáticas Aplicadas à Agricultura, Cidade Universitária Zeferino Vaz, s/n, \\ 13083-970 Campinas (SP), Brasil. \\ (*) Autora correspondente: camila.dourado@feagri.unicamp.br
}

Recebido: 23/abr./2013; Aceito: 5/jun./2013

\begin{abstract}
Resumo
O objetivo deste trabalho foi identificar zonas pluviometricamente homogêneas no Estado da Bahia e analisar as condições climáticas de cada zona entre 1981 e 2010. Foi aplicada a técnica de mineração de dados, Clusterização (agrupamento de dados), por meio do uso do algoritmo k-means, para transformação das séries históricas de precipitação em cinco zonas pluviometricamente homogêneas, em resposta à orografia, maritimidade e sistemas meteorológicos atuantes na região. Foram utilizados dados de médias mensais de precipitação de 92 estações meteorológicas. Os resultados apontam que as zonas mais secas estão situadas na parte central, de norte a sul do estado, principalmente ao norte com os menores volumes anuais, em torno de $480 \mathrm{~mm}$. A zona localizada ao norte do estado é contrastante com a faixa litorânea, em que são observados os maiores volumes anuais de precipitação (1.380 mm aproximadamente). A alta variabilidade pluviométrica ocorre em quase todas as zonas, principalmente em duas do semiárido com coeficientes de variação (CV) iguais a 42 e 28\%. Diferencia-se dessa característica a zona pertencente à faixa litorânea, que apresenta regularidade de chuvas durante todo o ano e CV de 15\%. As estações chuvosas e secas estão bem definidas. Os valores de precipitação da estação chuvosa representam em torno de $81 \%$ dos totais anuais, com destaque para as zonas situadas no centro-oeste e oeste do estado, com 95 e 96\% dos totais anuais.
\end{abstract}

Palavras-chave: mineração de dados, clusterização, variabilidade pluviométrica.

\section{Analysis of rainfall homogeneous areas in time series of precipitation in the State of Bahia, Brazil}

\begin{abstract}
The aim of this study was to identify rainfall homogeneous areas in the State of Bahia, Brazil and analyze the climatic conditions of each area for the period between 1981 and 2010. It was applied a data mining technique, clustering (grouping of data), by using the k-means algorithm for transforming time series of precipitation in five rainfall homogeneous areas, in response to topography, maritime dimension, and weather systems operating in the region of study. Data of average monthly rainfall of 92 meteorological stations were used. The results indicate that the driest areas are situated in the central part of the state, from north to south, mainly in the north with the lowest annual volumes, around $480 \mathrm{~mm}$. The area located in the north of the state contrasts with that one located on the coast, where the largest volumes of annual rainfall of the study were observed (approximately $1.380 \mathrm{~mm}$ ). The high rainfall variability occurs in almost all areas, especially in two of those of semiarid ones with Coefficients of Variation (CV) reaching 42 and 28\%. This characteristic differs from the area belonging to the coastal area, which presents regular rainfall during all the year and a CV of 15\%. The rainy and dry seasons are well defined. Precipitation values of the rainy season accounts for about $81 \%$ of the annual total, with emphasis on the zones located in the central-west and west of the state with 95 and 96\% of the annual total.
\end{abstract}

Key words: data mining, clustering, rainfall variability.

\section{INTRODUÇÃO}

As condiçóes climáticas, suas variaçôes e flutuaçôes, têm sido motivos do aumento de pesquisas científicas que visam à quantificação e o monitoramento mais preciso de variáveis ambientais. As variaçōes da distribuição espaço-temporal das chuvas, associadas aos baixos totais anuais pluviométricos, impactam diretamente a agricultura de sequeiro. O conhecimento dessa variável agrometeorológica pode orientar a tomada de decisão sobre as medidas necessárias para minimizar danos (Romani et al., 2010). 
Em particular, o Estado da Bahia, caracterizado por uma diversidade de domínios climáticos, apresenta um quadro de alta variabilidade pluviométrica, decorrente da conjunção de diferentes sistemas meteorológicos que atuam nessa região, tais como, Zona de Convergência Intertropical (ZCIT), Vórtices Ciclônicos (VCAN), Sistemas Frontais (FPA), Zona de Convergência do Atlântico Sul (ZCAS), brisas marítimas/terrestres e ventos vale/montanha, e um relevo constituído por planícies, vales, serras e montanhas (Molion e Bernardo, 2002; Cavalcanti et al., 2009).

O estado apresenta cinco tipos climáticos distribuídos nos sentidos Norte-Sul e Leste-Oeste, conforme a classificaçáo de THORNTHWAIte e Mather (1955), que dão origem às zonas árida, semiárida, subúmida a seca, subúmida a úmida e úmida. Os totais médios anuais da precipitação pluvial variam de 300 a $2.000 \mathrm{~mm}$, podendo ainda ultrapassar valores de $2.000 \mathrm{~mm}$ em algumas regiôes de clima úmido (Kousky, 1979).

A distribuição pluvial decrescente dos totais médios anuais, das extremidades Leste (Planície Litorânea) e Oeste (Chapadóes Ocidentais) para o interior semiárido, confirma a ação periférica dos sistemas dinâmicos. A Chapada Diamantina representa com maior expressividade o efeito orográfico, proporcionando um clima local no semiárido com chuvas anuais acima de $1.000 \mathrm{~mm}$ (TANAJUra et al., 2010). Tais características possibilitam a existência de regimes pluviais diferentes sobre o estado.

A pluralidade de regimes pluviais vinculada ao aumento da variabilidade interanual da precipitaçáo (SiLva et al., 2012) mostra a necessidade de sistemas que consigam analisar, com confiabilidade, informaçôes sobre a climatologia do estado.

Desta forma, a aplicação de técnicas de mineração, etapa principal do processo de Descoberta de Conhecimento em Bases de Dados (Knowledge Discovery in Databases - KDD) surge como uma alternativa promissora para transformar grandes volumes de dados climáticos em conhecimento novo e útil (FAYYAD et al., 1996; ; Han et al., 2011).

A escolha de uma técnica de mineração exige análise detalhada do problema em questáo e a decisáo sobre o tipo de padrão que será encontrado nos dados. Em particular, a Clusterização é uma técnica capaz de transformar séries históricas de dados pluviais em zonas homogeneamente pluviométricas. Cada posto meteorológico corresponde a uma série temporal de dados de uma cidade, enquanto uma região homogênea é formada pelo agrupamento das séries temporais mais similares.

Muitos trabalhos nas áreas de agrometeorologia e climatologia enfatizam a aplicação de clusterização para a definição de regiôes climáticas homogêneas em várias partes do Brasil. Abordado nos trabalhos de André et al. (2008) para o Rio de Janeiro, Fechine e Galvincio (2008) para Pernambuco e Boschi, Oliveira e Assad (2011) para o Rio Grande do Sul.
Neste contexto, o objetivo deste trabalho foi identificar zonas pluviometricamente homogêneas no estado da Bahia, e analisar as condiçôes climáticas de cada zona para o período de 1981 a 2010.

\section{MATERIAL E MÉTODOS}

A área de estudo corresponde ao estado da Bahia, com limítrofe ao Norte o rio São Francisco, no município de Curaçá, divisa com Pernambuco, entre as coordenadas 8032'00"S e 39॰22'49"W. Ao sul, o limite extremo é a Barra do Riacho Doce, no município de Mucuri, divisa com o Espírito Santo, 18²0’07”S e 39³9’48”W. A Leste, o ponto extremo é a Barra do Rio Real, no município de Jandaíra, divisa com o Oceano Atlântico, 11²7’07”S e 37020'37'W. O ponto extremo do Oeste é o divisor de águas, no município de Formosa do Rio Preto, divisa com o Tocantins, $1^{\circ} 17^{\prime} 21^{\prime \prime}$ S e 46 $36^{\prime} 54^{\prime \prime}$ W.

A metodologia aplicada para execução do processo de KDD, teve como suporte as etapas do modelo CRISP-DM (CRoss Industry Standard Process for Data Mining) descrita por Chapman et al. (2000). Esta metodologia é amplamente adotada em projetos de mineração de dados para definição dos procedimentos (Boschi et al., 2011; BнAкTikul et al., 2012). Neste trabalho, foram aplicadas quatro etapas do modelo CRISP-DM: entendimento dos dados, preparação dos dados, modelagem e avaliação.

$\mathrm{Na}$ primeira etapa (entendimento dos dados), foram utilizadas séries históricas de precipitação pluviométrica diária, adquiridas no sistema de informaçōes hidrológicas Hidroweb da Agência Nacional de Água (http://hidroweb. ana.gov.br/) (ANA, 2011). Optou-se por trabalhar com um período amostral de 30 anos, segundo padronizaçáo da Organizaçáo Meteorológica Mundial, para caracterizar o clima de uma regiáo. Sendo assim, foram selecionadas 92 estaçóes meteorológicas para os anos de 1981 a 2010, cobrindo praticamente todo o estado.

Foram utilizados dados consistidos, uma vez que dados brutos provenientes de estaçóes meteorológicas apresentam problemas, a saber, erros de leitura, transcrição, digitação e ausência de dados em alguns períodos. A consistência dos dados adotada pela ANA é baseada no modelo matemático proposto pela Agência Nacional de Energia Elétrica ANEEL (BRASIL, 1984).

Para implantaçáo dos dados espaciais, como por exemplo confecção dos mapas de localização das estações meteorológicas (Figura 1), distribuição pluviométrica anual e identificação das zonas homogêneas, foi adotado o sistema de coordenadas Latitude e Longitude, Datum SAD69, e sistema de projeção Universal Transversa de Mercator. As informaçôes geoespaciais foram fornecidas pela ANA (2011) e o shape, referente aos limites municipais do Estado da Bahia, pelo IBGE (2012). 
Para confecção do mapa pluviométrico da Bahia, foi aplicado o método geoestático IDW (Inverse Distance Weighted Ponderação do Inverso da Distância), que, de acordo com Marcuzzo, Andrade e Melo, (2011), é uma das técnicas de interpolação mais usadas para pontos espalhados espacialmente. Para isto, foram utilizadas as médias anuais de precipitação pluviométrica referente ao período de 1981 a 2010.

Para elaboração do cálculo da interpolação do valor de um ponto com base no método do IDW, foi utilizada a seguinte equação matemática Marcuzzo, Andrade e Melo (2011):

$Z(X)=\frac{\sum_{i=1}^{n} \omega_{i} Z(X)}{\sum_{i=1}^{n} \omega_{i}}$

Em que, $\mathrm{Z}(\mathrm{X})$ é o valor do ponto que se deseja interpolar; $\mathrm{n}$ é a quantidade de pontos próximos utilizados na interpolação do ponto $\mathrm{X} ; \mathrm{Z}\left(\mathrm{X}_{\mathrm{i}}\right)$ é o valor do ponto $\mathrm{X}_{\mathrm{i}}$; e $\omega_{\mathrm{i}}$ é o peso do valor de $\mathrm{X}_{\mathrm{i}}$ sobre o ponto $\mathrm{X}$. Para se determinar $\omega_{\mathrm{i}}$, foi utilizada a seguinte equação:

$\omega_{i}=\frac{1}{h(X, X)^{p}}$

Sendo, $\mathrm{h}\left(\mathrm{X}, \mathrm{X}_{\mathrm{i}}\right)$ a distância entre o ponto $\mathrm{X}$ e o ponto $\mathrm{X}_{\mathrm{i}}$; e p o parâmetro de potência, igual a dois.

A fase de preparação dos dados teve início com a construção de uma planilha composta por 92 linhas (registros), condizentes às estaçóes meteorológicas, e 10.957 colunas (atributos),

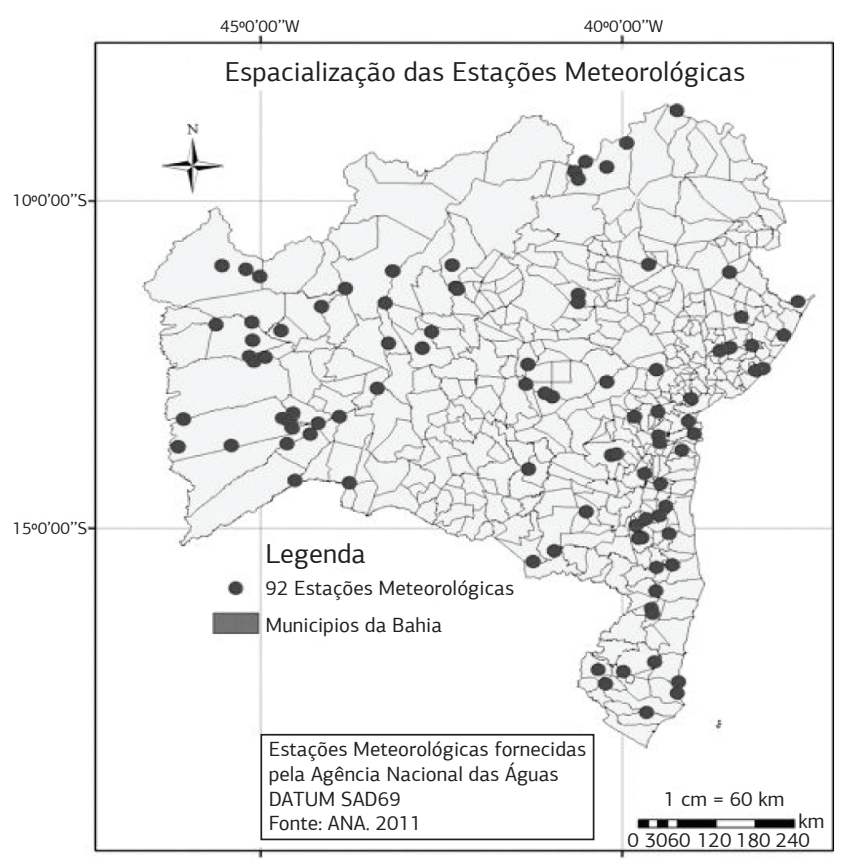

Figura 1. Espacialização das estaçôes meteorológicas do Estado da Bahia. relacionadas aos valores diários de precipitação. A disposição dos dias nas colunas e das estaçóes nas linhas foi organizada com o intuito de subsidiar a geração de agrupamentos das estaçóes meteorológicas conforme a similaridade de padrôes pluviais.

Com dados em escala diária, foi construído outro banco de dados com valores de precipitaçáo acumulados em escala mensal. Essa transformação foi necessária devido à particularidade de alta variabilidade da variável estudada, visto que a técnica de agrupamento busca padróes de similaridade entre os objetos para formação dos clusters.

Uma etapa essencial na aplicação das técnicas de clusterização, quando se utiliza atributos com diferentes ordens de grandeza, é a normalização dos dados. No entanto, para o presente trabalho esta etapa náo foi necessária, uma vez que o único atributo considerado foi a precipitação pluviométrica mensal, em que todos os acumulados mensais têm a mesma distribuição de dados.

$\mathrm{Na}$ etapa da modelagem, a técnica escolhida para seleção das zonas homogêneas foi a análise de clusters (agrupamento de dados), com o método de particionamento. Esta técnica consiste em agrupar objetos (itens de dados) que contenham características similares, ou seja, particionar uma dada populaçáo de objetos ou itens em conjuntos similares (Kumar et al., 2009) A similaridade entre objetos (séries temporais) é medida pela distância Euclideana entre dois objetos (Han et al., 2011). Quanto menor for a distância entre dois objetos, maior será a similaridade entre eles. Para análise de clusters, foi adotado o método de particionamento utilizando o algoritmo k-means. O programa computacional utilizado nesta fase foi o WEKA versão 3.6.3. (Witten et al., 2011).

No que se refere à metodologia de particionamento, de acordo com Rezende et al. (2005), os objetos são alocados nos clusters conforme a medida de similaridade adotada, onde se busca a maximizaçáo da distância entre clusters e a minimização da distância entre os objetos do mesmo grupo. O agrupamento ocorre com a especificação de uma medida de distância de acordo com o algoritmo, conforme a representatividade dos dados, ou quantitativa ou qualitativa.

Foram testados valores para $\mathrm{k}$ (n. ${ }^{\circ}$ de grupos $)=2,3,4$ e 5 para definição do número de zonas homogêneas mais condizente com a realidade dos dados. A escolha desse intervalo deu-se com base em trabalhos já realizados sobre a distribuição da precipitação pluvial da região e comparação com o mapa da pluviometria anual (Molion e Bernardo, 2002; Cavalcanti et al., 2009).

A última etapa aplicada do modelo CRISP-DM foi a avaliação. Nesta etapa foram discutidos os resultados obtidos, com a definição do número de clusters e identificação dos mesmos. Para uma melhor visualização dos resultados, os clusters foram espacializados, considerando-se a latitude e longitude de suas respectivas estaçóes. Nesta etapa do processo, a participação de especialistas do domínio foi fundamental para a escolha do melhor número de zonas homogêneas. 


\section{RESULTADOS E DISCUSSÃO}

A representação geoespacial da distribuição pluviométrica anual no Estado da Bahia, por meio do método geoestatístico IDW, permitiu uma melhor visualização da disparidade do volume pluvial existente no território baiano (Figura 2).

A aplicação da técnica de agrupamento permitiu a identificação das diferentes regiốes pluviométricas do estado da Bahia, de acordo com a similaridade do volume pluvial, calculada por meio da distância Euclideana entre cada par de séries temporais (Figura 3). Para esta etapa do trabalho, foi adotado como padrão do estudo a escala mensal do banco de dados.

Optou-se pela divisão de cinco zonas pluviometricamente homogêneas, uma vez que este número está em consonância com pareceres de especialistas da área e registros na literatura referentes à distribuição, a orografia, a maritimidade e aos sistemas pluviométricos que atingem o estado (Cavalcanti et al., 2009; Silva et al., 2012).

Os resultados mostram que o algoritmo k-means gerou os clusters de forma coerente com a distribuição pluviométrica espaço temporal do estado da Bahia. Resultados semelhantes foram encontrados ao comparar com o mapa pluviométrico do estado referente ao período de 1981 a 2010, obtido por meio da técnica de interpolação IDW (Figura 2).

Métodos de agrupamentos foram utilizados em vários trabalhos, em diferentes partes do Brasil, tais como: Alagoas (Souza et al., 1992), Pernambuco (Fechine et al., 2008), Rio de Janeiro (ANDré et al., 2008) e Rio Grande do Sul (Boschi et al., 2011).

De forma semelhante aos trabalhos de ANDRÉ et al. (2008), que utilizaram o k-means em dados meteorológicos do estado do Rio de Janeiro para identificar regiôes pluviometricamente homogêneas, sob forte influência dos fatores maritimidade e orografia, a delimitação das zonas homogêneas no estado da Bahia também apresentou fortes influências desses fatores climáticos.

Observa-se que a disposição dos clusters no território baiano evidencia a distribuiçáo decrescente nos volumes de chuva, das extremidades Leste (Planície Litorânea) e Oeste (Chapadóes Ocidentais) para o interior do estado da Bahia. Desta forma, confirma a ação periférica dos sistemas dinâmicos ZCIT, FPA, ZCAS e VCAN, que possibilitam a existência de regimes pluviais diferentes e superpostos sobre o território baiano, verificados da mesma forma por Cavalcanti et al. (2009).

O cluster 0 , representado por seis estações meteorológicas, situa-se ao Norte do estado. Os municípios deste grupo pertencem à região do semiárido nordestino, submetidos aos fortes rigores do clima árido, acometidos por longos períodos de estiagem, elevadas temperaturas, altas taxas de evapotranspiração e alta disponibilidade energética de luz solar (TeiXeIra et al., 2010). Sobre este cluster, atuam a Zona de Convergência Inter Tropical e os Vórtices Ciclônicos (Molion e Bernardo, 2002).

O cluster 1, representado por 21 estaçóes meteorológicas, abrange a regiáo mais setentrional, expandindo-se por toda parte central do Norte ao Sul do estado. Ocupa a maior área do território baiano, onde estáo localizados os municípios do semiárido pertencentes ao Polígono das Secas. São responsáveis pela distribuição pluviométrica deste grupo os VCAN, ao Norte, e a FPA nas demais áreas.

O cluster 2, composto por 21 estaçôes meteorológicas, delimita a região de transição do semiárido para o cerrado do Oeste baiano. Os municípios pertencentes a este grupo não são contíguos geograficamente como nos demais clusters, isto ocorre devido à expressividade do

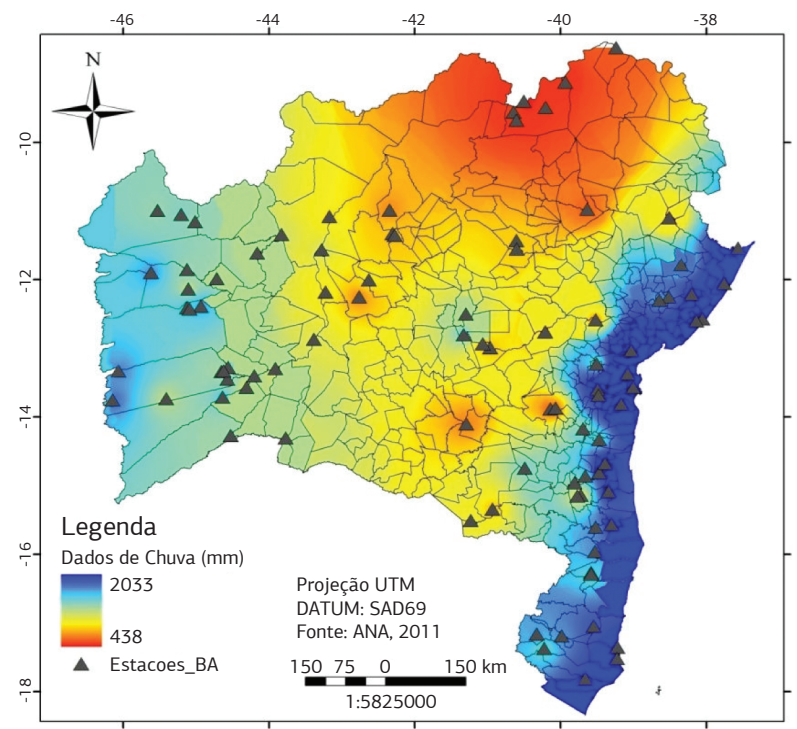

Figura 2. Mapa pluviométrico do Estado da Bahia - 1981 a 2010.

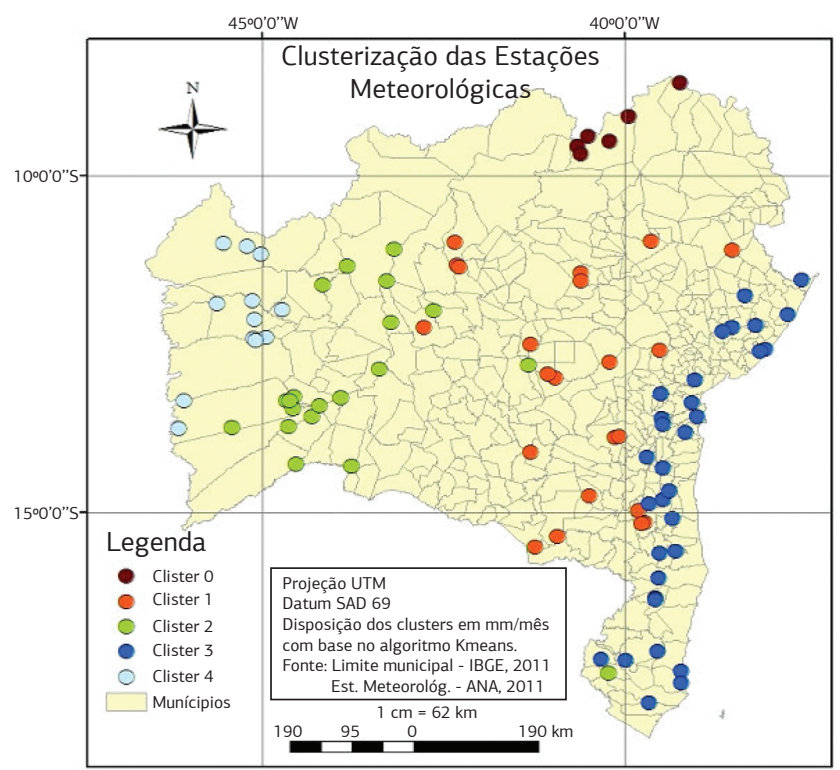

Figura 3. Agrupamento das zonas pluviometricamente homogêneas da Bahia. 
efeito orográfico da Chapada Diamantina, formando um micro clima no interior da região semiárida. Outro fator é a presença dos mecanismos de ação da FPA filiados ao regime climático da regiâo Sudeste do país, que influenciam o volume pluviométrico do Sudeste e Sul do estado (Cavalcanti et al., 2009).

O cluster 3, composto por 32 estaçóes meteorológicas, é representado pelos municípios da costa litorânea do estado, apresentando grande volume de chuva e relativa regularidade pluvial durante todo o ano. Sobre este cluster atuam os sistemas meteorológicos de FPA e as brisas marítimas. Representa a zona com maior índice pluviométrico, tal característica atribui a esta região a condição de baixo risco de seca, segundo os estudos de Risco de Seca do estado da Bahia realizado pela SEI (2000).

Por fim, o cluster 4, representado por 12 estaçôes meteorológicas, corresponde à parte ocidental do Oeste da Bahia, com períodos chuvosos e secos bem definidos ao longo do ano. É a região do cerrado baiano cuja concentração das atividades agropecuárias proporciona aos municípios desse grupo o maior PIB agrícola do Estado da Bahia. Possui o segundo maior volume pluviométrico e, sobre este grupo, atuam os Sistemas Frontais e a Zona de Convergência do Atlântico Sul.

Houve alta heterogeneidade na distribuição espacial das chuvas no estado, com precipitação média variando de $477 \mathrm{~mm}$ (cluster 0$)$ a $1.377 \mathrm{~mm}$ (cluster 3$)$ entre zonas pluviométricas (Tabela 1, Figura 4). Destaca-se uma diferença de $900 \mathrm{~mm}$ de chuva na média anual entre os clusters 0 e 3. A disposição espacial dos valores da variação pluviométrica corrobora com os estudos da SEI (2000) sobre a distribuição pluviométrica, apresentando maiores volumes de

Tabela 1. Valores acumulados anuais de precipitaçóes, médias, desvio-padrão, coeficiente de variação, máximo e mínimo para o período de 1981 a 2010

\begin{tabular}{|lcccccc}
\hline Cluster & Média & DP & CV (\%) & Mediana & Máximo & Mínimo \\
\hline 0 & 477 & 201 & 42 & 465 & 1148 & 160 \\
\hline 1 & 672 & 189 & 28 & 664 & 1147 & 323 \\
\hline 2 & 873 & 210 & 24 & 886 & 1337 & 512 \\
\hline 3 & 1377 & 209 & 15 & 1378 & 1861 & 900 \\
\hline 4 & 1059 & 219 & 21 & 1034 & 1531 & 695 \\
\hline
\end{tabular}

DP: desvio-padrăo; CV: coeficiente de variaçăo.

Tabela 2. Contribuiçấo da precipitação nos períodos chuvosos e secos para precipitação anual

\begin{tabular}{lccccc} 
& Cluster 0 & Cluster 1 & Cluster 2 & Cluster 3 & Cluster 4 \\
$\begin{array}{l}\text { Prec. Anual } \\
\text { (mm) }\end{array}$ & 477 & 672 & 873 & 1377 & 1059 \\
$\begin{array}{l}\text { Prec. Chuvoso } \\
(\mathrm{mm})\end{array}$ & 432 & 544 & 828 & 918 & 1014 \\
$\begin{array}{l}\text { Prec. Seco } \\
\text { (mm) }\end{array}$ & 45 & 127 & 45 & 459 & 45 \\
$\begin{array}{l}\text { \% Per. } \\
\text { Chuvoso }\end{array}$ & 90 & 81 & 95 & 66 & 96 \\
\hline$\%$ Per. Seco & 10 & 19 & 5 & 34 & 4 \\
\hline
\end{tabular}

chuva nas extremidades em direção ao interior do estado. Os clusters 0 e 1 apresentam os menores volumes pluviométricos e os maiores coeficientes de variação $42 \%$ e $28 \%$, respectivamente (Tabela 1), o que evidencia as características climáticas das regióes semiáridas, vistas também por SiLva, Pereira e Almeida (2012), ao estudar a variabilidade pluviométrica no semiárido do Nordeste brasileiro.

Já o cluster 3 apresenta o menor coeficiente de variação (15\%), representando a regiáo de maior altura e regularidade pluviométrica durante toda a série histórica. Observação semelhante encontra-se em Tanajura, Genz e Araújo (2010). Nos clusters 2 e 4, o coeficiente de variação corresponde a 24 e $21 \%$, respectivamente, indicando uma baixa variação no volume pluviométrico. $\mathrm{O}$ cluster 2 corresponde à regiáo mais próxima do semiárido, o que representa comportamento de variabilidade mais elevado em relação ao cluster 4, que faz parte de uma regiáo de cerrado e o segundo maior em níveis pluviométricos anuais.

A figura 5 mostra as distribuiçóes mensais da precipitação média de cada cluster determinada pelo algoritmo k-means. Desta forma, é possível comparar as regióes avaliando onde há maior ou menor variabilidade da média entre os clusters.

Observa-se que, durante a estação chuvosa, para os clusters $0,1,2$ e 4 há uma considerável dispersão entre os valores mensais. Entretanto, no período seco, apenas o cluster 1 apresenta variaçóes consideráveis em relaçáo aos demais. Verifica-se que a estaçáo chuvosa do cluster 3 é composta por outros meses em relação aos demais clusters.

A estação chuvosa do cluster 0 compreende aos meses de dezembro a abril, com volume máximo no mês de março (Figura 5). Isso ocorre devido à influência da ZCIT, que atinge a região nesse período, observado também por Kousky (1979) e CavalCanti (2009). A precipitação na estação chuvosa deste cluster é 90\% do total anual, caracterizando uma regiáo de estaçôes bem definidas ao longo do ano (Tabela 2). Compartilha desta mesma característica os clusters 2 e 4 , que apresentam no período chuvoso um total da precipitação anual de 95 e 96\%, respectivamente (Tabela 2). Apresentam semelhança entre os meses que compreendem o período chuvoso, outubro a abril, com volumes máximos nos meses de novembro a dezembro (Figura 5). O cluster 4 representa o grupo com o maior volume pluvial no período chuvoso em relação aos demais, alcançando um total superior a $1.000 \mathrm{~mm}$.

Observa-se que os volumes pluviais do período chuvoso do cluster 1 correspondem a $81 \%$ da precipitação total. Os sistemas meteorológicos FPA, ZCAS e VCANs são responsáveis pela produção de chuvas nessa região e contribuem para ocorrência de chuvas entre os meses de novembro a abril, com máximo em dezembro.

A precipitação dos períodos chuvosos e secos do cluster 3 é bem elevada, com destaque para volumes acima de $459 \mathrm{~mm}$ na estação seca, revelando regularidade na distribuição pluviométrica durante todo o ano. Apresenta $66 \%$ do montante pluviométrico no período chuvoso em relação ao total das chuvas anuais (Tabela 2). Os meses que integram a estação 
chuvosa desta região são março a julho, novembro e dezembro, com volumes máximos nos meses de dezembro e abril.

Considerando a contribuição da precipitação para as estaçôes chuvosa e seca em cada cluster (Tabela 2), verifica-se que o Estado da Bahia apresenta estaçóes bem definidas e que o período chuvoso é responsável por grande parte da precipitação anual. Com destaque para os clusters 0,2 e 4 com precipitação média do período chuvoso acima de $800 \mathrm{~mm}$ (clusters 2 e 4); $432 \mathrm{~mm}$ (cluster 0 ) para estação chuvosa e com $45 \mathrm{~mm}$ para estaçáo seca (clusters 0,2 e 4).

(a)

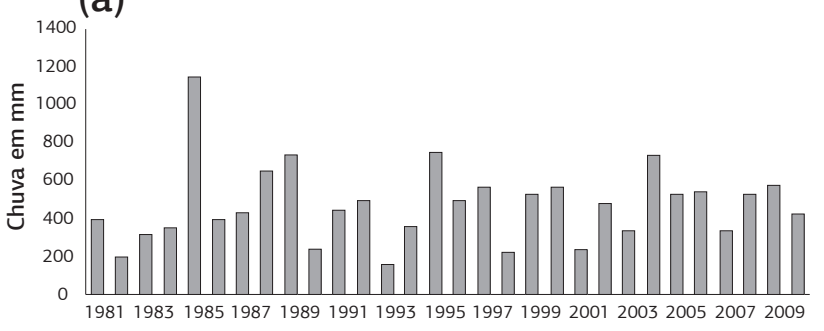

(b)

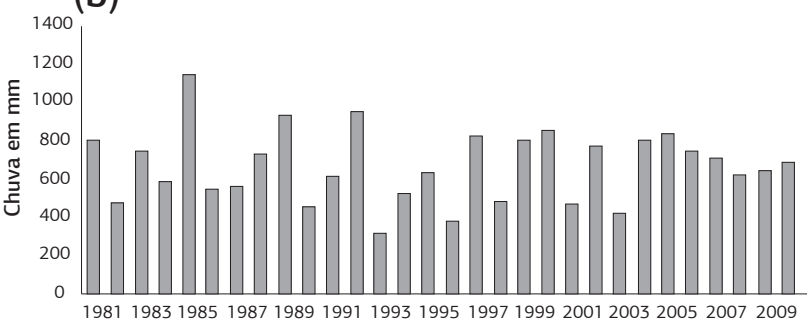

(c)

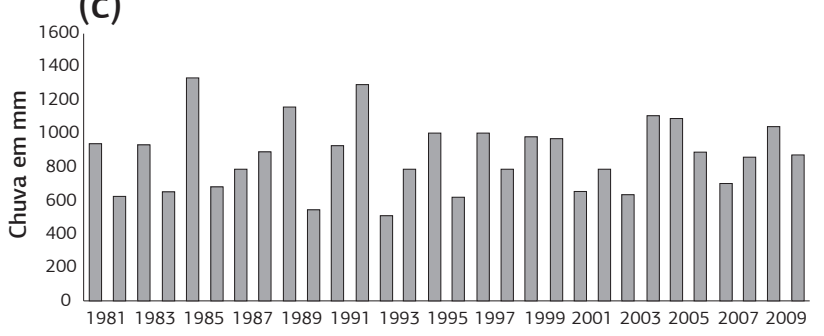

(d)

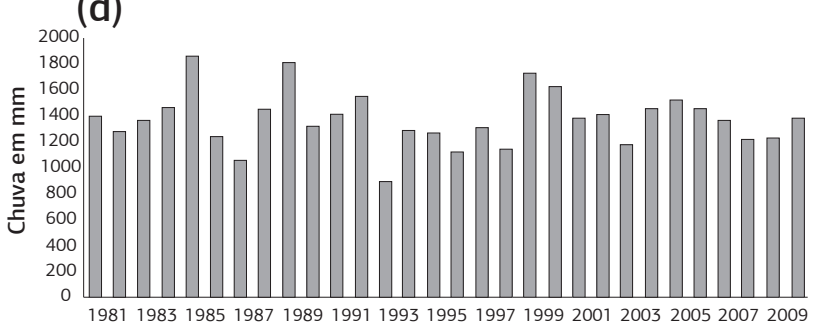

(e)

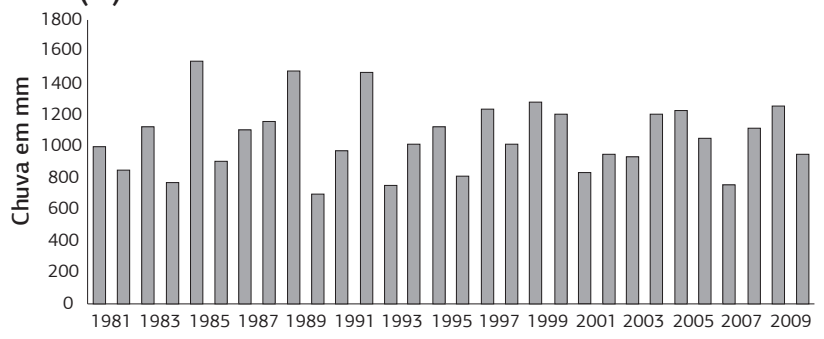

Figura 4. (a-e) Distribuição da precipitação pluviométrica nos cinco clusters (0, 1, 2, 3 e 4), para o período de 1981 a 2010 do Estado da Bahia.

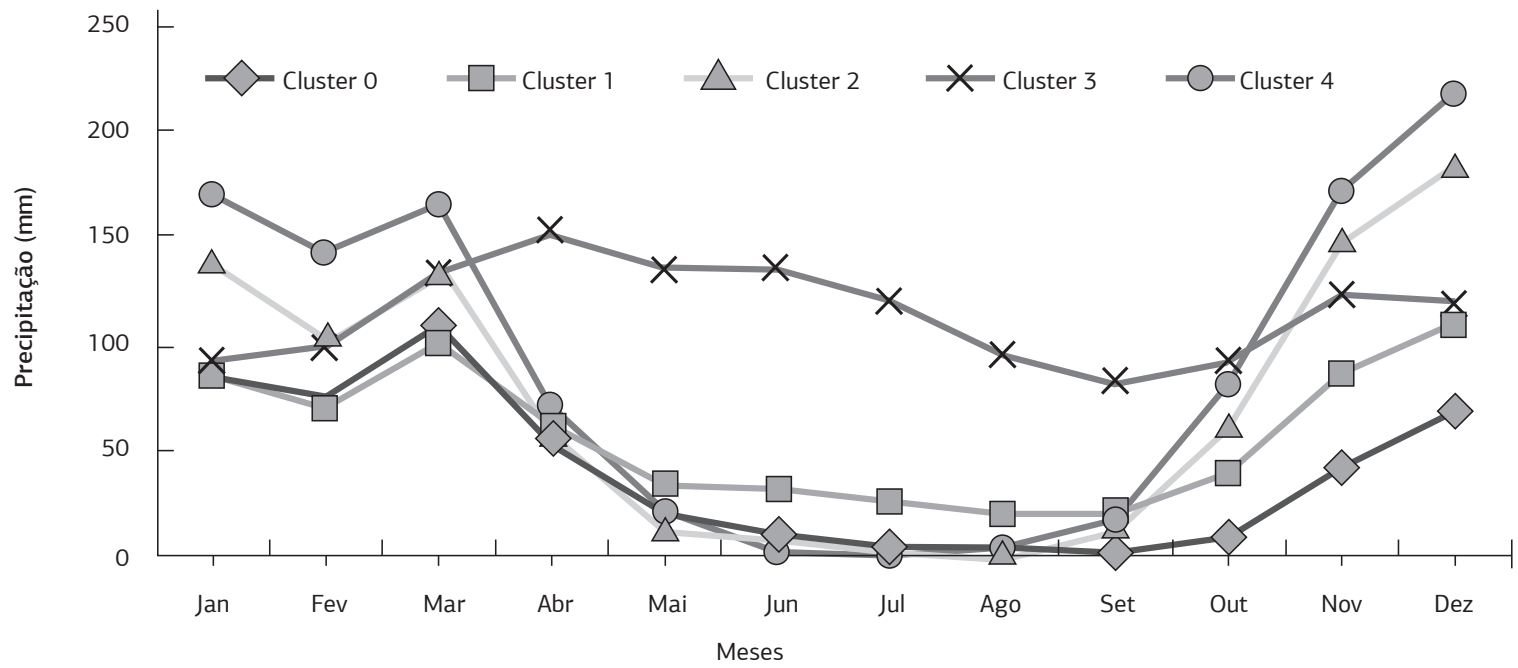

Figura 5. Precipitação média mensal em todos os clusters (0, 1, 2, 3 e 4) do Estado da Bahia, referente ao período de 1981 a 2010. 


\section{CONCLUSÃO}

Foram identificadas cinco zonas pluviometricamente homogêneas no Estado da Bahia, com os dados de precipitação correspondente ao período de 1981 a 2010. A técnica de mineração de dados, análise de cluster (agrupamento de dados), delimitou com coerência as regiôes pluviométricas do estado, mostrando-se eficiente para esta aplicação. $\mathrm{O}$ cluster, situado no centro-oeste do estado, difere dos demais quanto à disposição geográfica dos seus municípios. Integra a este cluster municípios do centro do estado, região semiárida, o que pode estar associado à interferência da orografia da região da Chapada Diamantina. Os valores de acumulados anuais da precipitação apresentaram alta variabilidade na distribuição pluviométrica em todos os clusters. A maior variaçáo foi registrada nos clusters que correspondem ao semiárido baiano, que apresenta como característica climática alta variabilidade pluviométrica. A menor variaçấo foi registrada na faixa litorânea, que apresenta como característica a regularidade e altos volumes pluviais durante todo ano. Em todo estado, as estaçôes secas e chuvosas são bem definidas, sendo os volumes pluviométricos do período chuvoso responsáveis por grande parte dos totais anuais de precipitaçáo.

\section{REFERÊNCIAS}

ANA - Agência Nacional das Águas. Disponível em: <http://hidroweb.ana. gov.br>. Acesso em: ago. 2011.

ANDRÉ, R.G.B.; MARQUES, V.S.; PINHEIRO, F.M.A.; FERRAUDO, A.S. Identificaçáo de regióes pluviometricamente homogêneas no estado do Rio de Janeiro, utilizando valores mensais. Revista Brasileira de Meteorologia, v.23, p.501-509, 2008. DOI: 10.1590/S0102-77862008000400009

BHAKTIKUL, K.; ANUJIT, R.; TOIM, J. Estimation of crop coefficient of corn $\left(\mathrm{Kc}_{\text {corn }}\right)$ under climate change scenarios using data mining technique. Environment Asia, v.5, p.56-62, 2012.

BOSCHI, R.S.; OLIVEIRA, S.R.M.; ASSAD, E.D. Técnicas de mineraçáo de dados para análise pluvial decenal do Rio Grande do Sul. Engenharia Agrícola, v.31, p.1189-1201, 2011. DOI: 10.1590/S010069162011000600016

BRASIL. Departamento Nacional de Águas e Energia Elétrica - DNAEE. Divisão de Controle de Recursos Hídricos. Sistemática para análise de consistência e homogeneização de dados pluviométricos, Brasília, 1984.

CAVALCANTI, I.F.A.; FERREIRA, N.J.; DIAS, M.A.F.; JUSTI, M.G.A. Terra e clima no Brasil. São Paulo: Editora Oficina de Textos, p.464, 2009.

CHAPMAN, P.; CLINTON, J.; KERBER, R.; KHABAZA, T.; REINARTZ, T.; SHEARER, C.; WIRTH, R. CRISP-DM 1.0: step-bystep data mining guide. Illinois: SPSS, p.78, 2000.

FAYYAD, U.; PIATETSKY-SHAPIRO, G.; SMYTH, P. From data mining to knowledge discovery: an overview. In: Advances in knowledge discovery and data mining, eds. U. M. Fayyad et al., AAAI/MIT Press. Menlo Park: American Association for Artificial Intelligence, Calif, pp. 1-34, 1996.
FECHINE, J.A.L.; GALVINCIO, J.D. Agrupamento da precipitaçáa mensal da bacia hidrográfica do rio Brígida-PE, através da multivariada. Revista Brasileira de Geografia Física, v.1, p.39-46, 2008.

HAN, J.; KAMBER, M. Data mining: concepts and techniques. San Francisco: Morgan Kaufmann Publishers, p.770, 2011.

IBGE- Instituto Brasileiro de Geografia e Estatística. Clima da Bahia. Disponível em: <www.ibge.gov.br>. Acesso em: jul. 2012.

KOUSKY, V.E. Frontal influences on northeast Brazil. São Paulo: INPE, p.16, 1979. DOI: 10.1175/1520-0493(1979)107<1140:FIONB>2.0.CO;2

KUMAR, D.N.; DHANYA, M.C.T. Data mining and its applications for modeling rainfall extremes. Journal of Hydraulic Engineering, v.15, p.2551, 2009. DOI: 10.1080/09715010.2009.10514967

MARCUZZO, F.FN; ANDRADE, L.R.; MELO, D.C.R. Métodos de interpolação matemática no mapeamento de chuvas no estado do Mato Grosso. Revista Brasileira de Geografia Física, v.4, p.793-804, 2011.

MOLION, L.C.B.; BERNARDO, S.O. Uma revisão da dinâmica das chuvas no nordeste brasileiro. Revista Brasileira de Meteorologia, v.17, p.1-10, 2002.

REZENDE, S.O.; PUGLIESI, J. B., MELANDA, E.A.; PAULA, M.F. Mineração de dados. In: Sistemas inteligentes. Fundamentos e aplicações. Barueri: Manole Ltda., p.527, 2005.

ROMANI, L.A.S.; ÁVILA, A.M.H.; ZULLO, J.J.; TRAINA, C.J.; TRAINA, A.J.M. Mining relevant and extreme patterns on climate time series with CLIPSMiner. Journal of Information and Data Management, v.1, p.245-260, 2010.

SILVA, V.P.R.; PEREIRA, E.R.R.;ALMEIDA, R.S.R. Estudo da variabilidade anual e intra-anual da precipitação na regiáo nordeste do Brasil. Revista Brasileira de Meteorologia, v.27, p.163-172, 2012. DOI: 10.1590/S0102-77862012000200005

SOUZA; J.L., AMORIM, R.F.C.; CARVALHO, S.M.R.; PEREIRA, J.O.; CURI, P.R.C. Agrupamento de estaçôes pluviométricas do estado de Alagoas, utilizando-se análise multivariada. Revista Brasileira de Meteorologia, v.7, p.603-612, 1992.

SUPERINTENDÊNCIADEESTUDOSECONÔMICOSESOCIAIS DA BAHIA (SEI). Sistemas de dados estatísticos. 2000. Disponível em: $<$ http://www.sei.ba.gov.br/side/consulta>. Acesso em: maio 2011.

TANAJURA, C.A.S.; GENZ, F; ARAÚJO, H.A. Mudanças climáticas e recursos hídricos na Bahia: validação da simulação do clima presente do HADRM3P e comparaçáo com os cenários A2 e B2 para 2070 - 2100. Revista Brasileira de Meteorologia, v.25, p.345-358, 2010. DOI: 10.1590/ S0102-77862010000300006

TEIXEIRA, A.H.C.; MOURA, M.S.B; ANGELOTTI, F. Cultivo da mangueira. EMBRAPA SEMIÁRIDO - Sistema de Produção, v.2, 2010.

THORNTHWAITE, C.W.; MATHER, J.R. The water balance. Climatology, v.8, p.1-40, 1955. DOI: 10.1097/00010694195904000-00024

WITTEN, I.H.; FRANK, E.; HALL, M.A. Data mining: practical machine learning tools and techniques. 3rd ed. San Francisco: Morgan Kaufmann, p.629, 2011. DOI: 10.1145/507338.507355 